\title{
WiMAX Cell Level Simulation Platform Based on ns-2 and DSP Integration
}

\author{
Adam Flizikowski, Rafał Kozik, Henryk Gierszal, Marcin Przybyszewski, and Witold Hołubowicz
}

\begin{abstract}
The WiMAX (Worldwide Interoperability for Microwave Access) system based on the IEEE 802.16 family of standards is a promising technology for last-mile access. Both IEEE 802.16 and 3GPP-LTE systems candidate for becoming the $4 \mathrm{G}$ network of choice. The need to evaluate multiple performance enhancing techniques like MIMO, OFDM(A), novel channel coding schemes like non-binary LDPC codes, together with development of standards like IEEE 802.21, that aims at enabling handover and interoperability between heterogeneous network types, make rapid prototyping-based simulations an important issue. This paper presents a novel approach to $4 \mathrm{G}$ oriented simulation environment that integrates popular network simulator (ns-2) and a Digital Signal Processing (DSP) to enable comprehensive link layer and cell level simulations. Proposed simulation environment is intended as an evaluation platform for assessing QoS/QoE and Connection Admission Control (CAC) algorithms designed for WiMAX systems. Moreover we study ways to improve simulation time (with focus on AWGN channel simulation) by using CUDA parallel processing technology for NVIDIA graphic cards.
\end{abstract}

Keywords-FEC codes, nb-LDPC, DSP, integrated platform, ns-2.

\section{INTRODUCTION}

$\mathbf{T}$ HE GOAL of IEEE 802.16 standardization efforts is to provide a comprehensive and accurate new generation broadband wireless standard specification. New generation of BWA networks are expected to be capable of handling hundreds of user connections over a wide area assuring high QoS for different user traffic. The requirement for IMTAdvanced systems are concerned by IEEE $802.16 \mathrm{~m}$ specification, since by 2010 it is assumed that such system will provide throughputs (three) higher than these of current IMT2000 systems $(30 \mathrm{Mb} / \mathrm{s})$ and depending on user mobility level, offer throughputs from $100 \mathrm{Mb} / \mathrm{s}$ for mobile users moving at high speed up to $1 \mathrm{~Gb} / \mathrm{s}$ for stationary solutions [1].

Moreover ITU-R defines User Plane Latency should be less than $10 \mathrm{~ms}$ in unloaded conditions (for small IP packets) for both downlink and uplink. In order to meet these strict requirements it may be necessary to define multiple radio interfaces (separately) for high and low-mobility users. In this light 3GPP-LTE and WiMAX seem best candidates to

The research leading to these results has received funds from the European Community's Seventh Framework Programme (FP7/2007-2013) under grant agreement no. 216203 (DaVinci project).

A. Flizikowski and M. Przybyszewski are with the ITTI Ltd., ul. Rubiez 46/C, 61-612 Poznan, Poland (e-mail: aflizikowski@itti.com.pl; mprzybyszewski@itti.com.pl).

R. Kozik is with the University of Technology and Life Sciences, Institute of Telecommunications, ul. Kaliskiego 7, 85-796 Bydgoszcz, Poland.

H. Gierszal and W. Hołubowicz are with the Adam Mickiewicz University Poznan, Poland (e-mail: holubowicz@amu.edu.pl). meet these requirements. Ongoing efforts to improve current systems performance focus on areas like channel coding effectiveness [13], optimizing H-ARQ techniques [12],[9], turbolike channel estimation [4], resource allocation with special focus on Connection Admission Control techniques (CAC) and QoS provisioning. This paper presents a novel simulation environment motivated by the need to evaluate $4 \mathrm{G}$ system equipped with both MAC and physical layer at the satisfactory level of fidelity and standard compliance. It is intended as an evaluation platform for multiple CAC algorithms designed for $4 \mathrm{G}$ networks. Studies leading to this research have been conducted as part of work within the FP7 DA VINCI project.

This paper is organized as follows. Section 2 describes the approach to QoS handling in WiMAX. In section 3 several simulating platforms and tools are compared against the accuracy of IEEE 802.16 standard implementation. Section 4 describes the motivation and details of the integrated ns-2/DSP platform. In section 5 the necessary platforms configuration procedures are described. Section 6 and 7 describe the platform validation and measurements results. The final section discusses pros and cons of proposed integrated platform.

\section{QOS CONCEPTS IN WIMAX}

The performance enhancing techniques and mechanisms located in physical layer include among all OFDM technology, logical and physical channels, adaptive modulation and coding, intelligent antennas, MIMO technology, error detection and correction and power control. On the other hand the IEEE 802.16 MAC layer is far more complex than its counterpart in WLAN (IEEE 802.11). The key part in the MAC processing is realized by the Common Part Sub-layer (CPS), one of the three MAC sub-layers defined in the standard. CPS enables provision of QoS parameters that in WiMAX depend on the variety of factors like residual bandwidth, fluctuating channel capacity due to changes in radio channel or terminal transmission capabilities that vary as a function of terminal location with respect to the serving base station (BS). In the IEEE specification the key resource management functions and admission control are located in the management plane (Fig. 1). The concept of guaranteeing QoS in 802.16 networks is based on a few mechanisms e.g. Admission Control, dynamic service management.

The IEEE 802.16 defines five classes of service in the CPS sub-layer [6], [7]. Classes are differentiated by means of QoS parameters. In order to deliver requested QoS to connections BS has to allocate enough resources, however it is out of scope of the standard to decide how such task 
Data/Control Plane

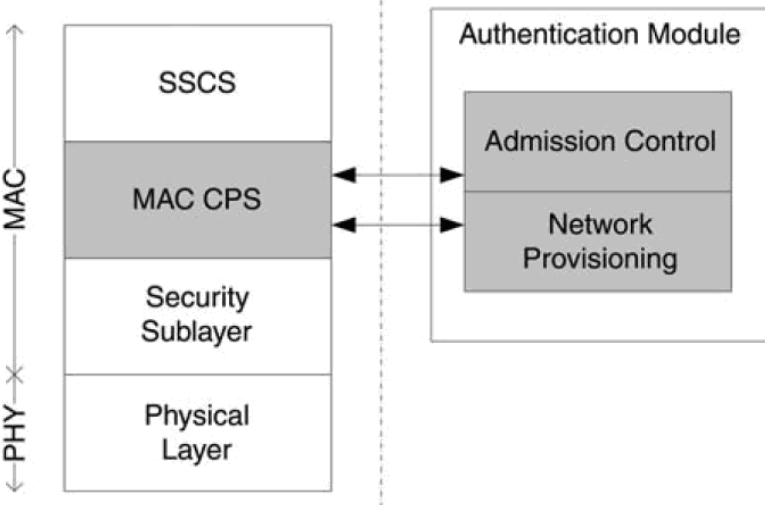

Fig. 1. QoS model in IEEE 802.16 [10].

is to be accomplished. The IEEE 802.16 specification does not define either admission control or - except for UGS uplink scheduling mechanisms for WiMAX. That is why the research on the aforementioned algorithms by simulations is so important.

\section{Simulation EnVIRONMENT}

Since we need accurate implementation of higher ISO/OSI stack layer's protocol (mainly application's and data link's layer) to proceed with cell level simulations, while at the same time we require accurate physical layer model to assess radio channel's innovations proposed by DaVinci project, the task of choosing an appropriate simulation platform (or platforms) becomes an important issue. Therefore in this section the open-source and commercial network simulators application and solutions are compared against accuracy of IEEE 802.16 standard implementation.

\section{A. Network Simulator $(n s-2)$}

Network Simulator is a widely used simulation tool that implements a variety of entities, such as wireless node (IEEE 802.11), wireless channel and protocols. Although by default ns-2 does not implement model of the IEEE 802.16 networks, a couple of 3rd party patches are available, that implement WiMAX with different precision. At the present moment we are aware of the following WiMAX modules:

- NIST module [10]

- NIST+ module [2]

- PMP module [3]

- LRC module [5]

- WINSE [11]

The first of the modules (NIST) delivers most comprehensive set of PHY layer blocks at the expense of MAC layer fidelity. Moreover, it does not implement service flows and there is no possibility to adjust parameters for QoS provisioning. It also lacks error correction mechanism. The PMP module does not support mobility (IEEE 802.16e) and it does not provide means for QoS provisioning however there are functions for processing CAC already implemented. Unlike NIST and PMP, LRC supports QoS features and implements five service flow classes compliant with the IEEE 802.16e standard. Still, with this WiMAX module it is impossible to configure modulation type and coding schemes. Proposed in [8] NIST+ is an interesting package extending functionality of original NIST module. It shares PHY properties with NIST module, adds QoS classes, QoS management, unicast and contention request opportunities mechanisms and scheduling algorithms for the UGS, rtPS and BE. NIST+ implements three most important service classes with unicast and contention request modes. Both NIST and NIST+ implement Dynamic Service Addition messages (DSA-x). Additional advantage of this patch is good documentation of the underlying NIST core part. What is also important, its source code is transparent and upgrade- friendly. WINSE described in [11] is another WiMAX extension for ns2. It supports both OFDM and OFDMA, implements ARQ and HARQ mechanisms and supports fragmentation and packing. Moreover it supports all five service flow classes defined in 802.16e. It is possible to configure modulation type and coding schemes. Although this module is the most advanced ns-2 WiMAX extension, it is being developed for Nokia and is not available on the web. When looking at the pros and cons of $\mathrm{ns}-2$, it is important to stress the fact that this environment is useful for cell-level evaluation, but mainly with respect to wired networks. The ns-2 simulation capabilities of L1L2 ISO/OSI layers with respect to DaVinci requirements have been presented in table (Table I).

\section{B. Matlab}

One of the major drawbacks of ns-2 WiMAX physical layer blocks is insufficient support for different channel models (AWGN, Rayleigh), limited availability of channel coding schemes (e.g. LDPC) and MIMO techniques. The Matlab/Simulink (Mathworks) offers by default much more solutions in this area. Default elements from the Simulink library meet most IEEE 802.16 PHY requirements. Simulink community provides a variety of custom blocks, so it is not unusual to find implementation of (binary/non-binary) LDPC codes and Turbo-Codes [11]. Moreover Matlab offers more advanced and sophisticated tools for radio characteristics analysis (modulation constellation analysis, automatic BER assessment, built-in radio calibration models). Although Simulink is well suited for PHY simulation, it is not intended for simulating higher ISO/OSI layers. As the authors are aiming at evaluation of the cell level performance of WiMAX, a lot of programming effort would have to be put in order to model MAC and higher layers in Matlab/Simulink. On the contrary the availability of higher-layer protocols libraries, agent-nodes and the ability to define trajectory of mobile terminals position ns-2 as the solution complementary to Matlab (with Communication Toolbox). Additional functionality builtin Matlab with respect to physical/link layer is essential when trying to asses/distinguish cell level performance according to requirements from [13] by means of simulation. The RealTime workshop (RTW), the Matlab extension package, enables automated code generation, compilation and consolidation for 
TABLE I

SuMMARY OF SIMULATORS CAPABILITIES WITH RESPECT TO THE DAVINCI REQUIREMENTS

\begin{tabular}{|c|c|c|c|c|c|}
\hline Block & $\begin{array}{c}\text { Matlab/Simulink } \\
\text { R2008b } \\
\end{array}$ & Patch 1 (NIST) & $\begin{array}{l}\text { Network simulator } \\
\text { Patch } 2 \text { (PMP) } \\
\end{array}$ & Patch 3 ( LRC) & Da Vinci requirements \\
\hline Modulation & $\begin{array}{c}\text { *BPSK, *QPSK } \\
\text { *4-QAM } \\
\text { *16-QAM } \\
\text { *64-QAM }\end{array}$ & $\begin{array}{l}* \text { BPSK, } \\
\text { *QPSK } \\
* 16-\text { QAM } \\
* 64-Q A M\end{array}$ & $\begin{array}{l}* \text { QPSK } \\
* 16-\text { QAM } \\
* 64-\text { QAM }\end{array}$ & no data & $\begin{array}{c}\text { *UL: QPSK and 16-QAM } \\
\text { *DL: QPSK, 16-QAM, } \\
\text { 64-QAM, 256-QAM, } \\
\text { BPSK }\end{array}$ \\
\hline OFDM / OFDMA & yes/yes & yes/no & yes/no & yes/no & yes/yes \\
\hline Coding & $\begin{array}{l}\text { *Convolutional Coding } \\
\text { *Turbo Coding } \\
\text { *Convolutional Turbo } \\
\text { Coding } \\
\text { *LDPC }\end{array}$ & no data & no data & no data & $\begin{array}{c}\text { *Convolutional } \\
\text { Turbo Coding } \\
\text { *Convolutional Coding } \\
\text { *LDPC, NB-LDPC, } \\
\text { NB-root-LDPC }\end{array}$ \\
\hline $\begin{array}{c}\text { Channel } \\
\text { (Propagation } \\
\text { model) }\end{array}$ & $\begin{array}{c}* \text { AWGN } \\
\text { * Rayleigh (no fading, } \\
\text { flat-fading, } \\
\text { selective fading) } \\
\text { *Doppler spread }\end{array}$ & $\begin{array}{c}\text { *free space }(\mathrm{FS}) \\
\text { model } \\
\text { *two ray ground } \\
(\mathrm{TRG}) \text { model } \\
\text { *shadowing } \\
\text { model }\end{array}$ & $\begin{array}{c}* \text { free space }(\mathrm{FS}) \\
\text { model } \\
\text { *two ray ground } \\
(\mathrm{TRG}) \text { model } \\
\text { *shadowing } \\
\text { model }\end{array}$ & $\begin{array}{c}\text { *free space }(\mathrm{FS}) \\
\text { model } \\
\text { *two ray ground } \\
(\mathrm{TRG}) \text { model } \\
\text { *shadowing } \\
\text { model }\end{array}$ & $\begin{array}{c}* \text { AWGN } \\
* \text { Rayleigh }\end{array}$ \\
\hline MIMO & $2 \times 1,2 \times 2$ & No & no & no & at least $2 \times 1$ (typical: $2 \times 2$ ) \\
\hline Coding rates & $1 / 2,2 / 3,3 / 4,5 / 6$ & $1 / 2,3 / 4$ & $1 / 2,3 / 4$ & no data & $1 / 2,2 / 3,3 / 45 / 6$ \\
\hline
\end{tabular}

a target DSP board (e.g. TI DSK 6000) based on Simulink model. Thus assuming a fixed-point hardware-oriented implementation of required PHY blocks this option enables interesting DSP deployment capabilities. Thanks to advanced profiling features it is possible to assess each block's computational complexity.

\section{IMPLEMENTATION OF INTEGRATED PLATFORM}

Functional complementarity of both aforementioned simulation environments (ns-2, Matlab/DSP) in the area of IEEE 802.16 standard compliance creates an opportunity to merge their virtues by the means of an integrated platform. The idea of proposed integration solution has been presented in the figure (Fig. 2). The key enabler of this solution is a physical Ethernet link between the MAC layer implemented in ns-2 and WiMAX PHY blocks designed in Matlab and deployed on a target DSP board.

One of the biggest advantages of using Matlab as layer between the DSPs IDE called Code Composer Studio (CCS) and developer is that it allows moving forward from hand code writing to high level model-based design. Model-Based design makes it easy to express a design concept, simulate

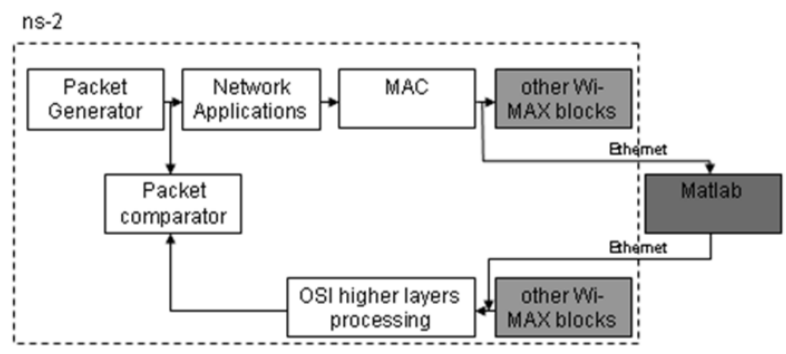

Fig. 2. Integrated ns-2, Matlab/DSP platform concept. the model to verify algorithms, automatically generate and deploy code on a hardware target, and verify exactly the same operation on silicon. Moreover it is possible to implement the design on a Texas Instruments DSP, and verify its on-target performance in real time, simply by adding dedicated block (Target Preferences Block) from target processor blockset library. The advantages of the integrated platform proposed herein stem from the following facts

- various high-level models can be easily deployed on DSP and integrated with ns-2 without a line of coding

- ease of modifying/deploying model updates (e.g. in Matlab/Simulink) brings the platform closer to the concept of rapid prototyping

- it is possible to use the DSP hardware-in-the-loop (HWIL) extension in real-time (emulation) or virtual time (simulation) mode

- in addition to the built-in Ethernet card, DSP board supports JTAG communication with host computer introducing real-time communication with the board

- single DSP can be used to model several nodes (multiuser) with slight (but generic) modifications to ns-2

- measuring real DSP processing delays, BER can be introduced and analyzed if needed

- with the model code optimized for target DSP (e.g. LDPC decoder) the integrated ns-2/DSP SLS platform can be used in real-time (emulation) mode; the DSP equipped with additional $\mathrm{HW}$ modules enables real RF communication

The key disadvantage of the solution using DSP in the loop of ns-2 simulator is that by default (e.g. without applying optimizations to the Simulink model) the DSP processing time of the deployed model is not suitable for real-time emulation model (only virtual-time simulation mode applies). 
TABLE II

BER CHARACTERISTICS FOR MATLAB Simulation COMPARED With LUT

\begin{tabular}{ccc}
\hline \hline EbNo & ns-2 & Matlab \\
\hline 8.25 & 0.9974 & 0.9974 \\
8.5 & 0.9948 & 0.9948 \\
8.75 & 0.9923 & 0.9922 \\
9,00 & 0.9897 & 0.9896 \\
9.25 & 0.9783 & 0.9782 \\
9.5 & 0.9557 & 0.9556 \\
9.75 & 0.9032 & 0.9031 \\
10,00 & 0.8104 & 0.8103 \\
10.25 & 0.6163 & 0.6163 \\
10.5 & 0.3958 & 0.3959 \\
10.75 & 0.2132 & 0.2131 \\
11,00 & 0.0976 & 0.0974 \\
11.25 & 0.0401 & 0.0401 \\
11.5 & 0.0162 & 0.0163 \\
11.75 & 0.0037 & 0.0037 \\
12,00 & 0.0008 & 0.0008 \\
20,00 & 0.0008 & 0.0008 \\
\hline \hline
\end{tabular}

\section{A. LUT Approach to Model Physical Layer}

Although approach integrating ns-2 and 802.16 PHY model deployed as Simulink model is interesting from the perspective of functional capabilities of the model, it can also be computationally demanding for simulations with large number of users, as Simulink channel is shared by all SS and BS nodes. Therefore the abstraction based on the idea of Look Up Tables (LUT) for simulation for various FEC codes (nbLDPC, LDPC, BTC) was used. The approach is shown in Fig. 3. The process is divided into two phases. In phase I the channels characteristics (EbNo vs. BER/WER/CWER) are gathered during Matlab simulation. Those are used by ns-2 in form of LUT tables to mimic MATLAB channel. As a result EbNo vs. BER/WER/CWER characteristics for LUT will be exactly the same as for Matlab simulation(Table II).

\section{Platforms Configuration}

In order to make full use of integrated platform some additional modifications concerning communication are required. Since there are 3 distinct integration approaches (Fig. 4) each of them requires specific preparation.

\section{A. Matlab/DSP Environment}

In this section major issues related to the connectivity of the DSP deployed model are discussed. With the use of Matlab

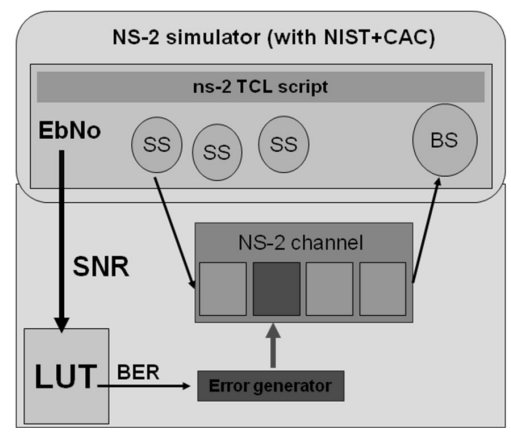

Fig. 3. Lookup table approach. extension package, namely the Instrument Control Toolbox (ICT), it is possible to connect Matlab model with an external (e.g. ns-2) server (see Host1 in the figure Fig. 5) in a simple way by means of TCP/IP communication.

The implementation using two optional network communication interfaces IA1 and IA2 (IEEE 802.3), that connect ns-2 and PHY model, has been presented in the figure (Fig.5). Duality of the interfaces indicates that it is possible to deploy/run model on both Matlab/Simulink environment and target DSP board (Texas Instruments DSK C6455) [13] either using relay PC (Host2) or directly communicating via Ethernet connection (IA2). The DSK C6455 kit is by default equipped with two communication interfaces: a) a $10 / 100 \mathrm{Mb} / \mathrm{s}$ Ethernet card and b) JTAG (IEEE 1149.1) USB emulator for real-time DSP CCS communication.

During first phase of development, our platform incorporated an older version of TI DSP board, namely DSK c6416. DSK c6416 did not have a built-in Ethernet interface, so Host2 had to act as a proxy during communication between ns-2 and DSP target board. However both floating-point computations typical for running Matlab models and non Real-Time operating system (WindowsXP) that introduces non-deterministic allocation of Host 2 processor's resources, resulted in poor performance and low stability of the physical layer model. Moreover sending data packets to/from WiMAX MAC layer (ns-2) from/to DSP module utilized RTDX (Real Time Data eXchange), an extension that enables real time tracing and debugging of a running program. It allows not only reading but also sending data to a program running on DSP processor. This approach benefits from small DSP load footprint during host target board communication. An interesting feature of the TI C6000 DSP Toolbox is that it provides a set of blocks dedicated (optimized) to perform computationally expensive calculations using processor-specific instructions set. The RF model functions that would benefit from this capability are among all FFT/IFFT, various matrix operations etc.

\section{B. $n s-2$ Nodes' Communication Enhancement}

The ns-2 introduces capabilities to support communication with external nodes. To the best authors knowledge there exist two methods to support external communication:

- running the ns-2 in emulation mode

- modifying current MAC PHY API to handle external communication with HW module.

When in emulation mode, the simulated nodes can by means of special objects called tap-agents network objects exchange

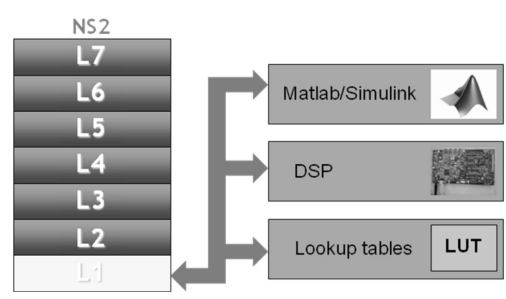

Fig. 4. Integration approaches. 


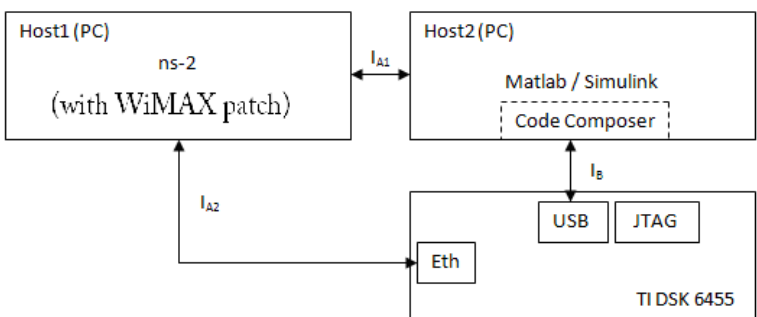

Fig. 5. Target platforms interfaces diagram.

packets with host computer (Host1) that is connected to a physical network [8] .

The other approach requires some additional programming effort, as it is necessary to modify simulator's source code. As a result it gives a better control over ns- 2 task scheduler and additional degrees of freedom when choosing the right ns-2 WiMAX module (patch). Changes made to generic wireless node were summarized in table (Table III).

\section{Look Up Tables Adaptation}

Enabling LUT table support in ns-2 platform required implantation changes in WirelessChannel class. The modifications are shown in diagram (Fig. 6). Switching between channel model types is transparent for the simulation and is set up using TCL script.

The errors generation process is based on the BER value maintained in LUT for particular EbNo and is shown in Fig 7.

\section{LINK LEVEL MODEL}

The link layer model used for preliminary tests of the combined ns-2/DSP platform includes AWGN channel with simple transmitter and receiver implementing the nb-LDPC

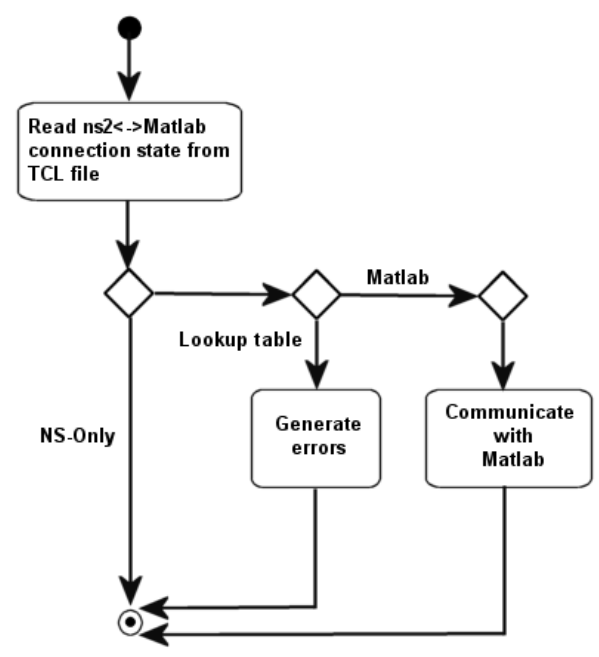

Fig. 6. Flexibility of link layer configuration (ns-2, lookup table, Matlab/DSP model). codes. The sequence of steps leading to fully operational simulation platform includes:

- take encoder/decoder source C file

- adapt it to Simulink (wrapping)

- verify and validate the model (against pure $\mathrm{C}$ results of the original, C-language nb-LDPC implementation)

- attach Ethernet Receiver/Sender to the Matlab/Simulink model

- deploy the resulting model on DSP

- verify and validate the effect of deployment

- make basic DSP measurements of the physical layer abstraction (BER, profiling, delay)

- connect DSP to ns-2 via Ethernet cable

- perform ns-2 cell level, end-to-end, measurements (virtual time simulation).

It is essential to underline that the above process of deploying the target physical layer blocks on the DSP is done with the following assumptions:

- no performance optimization of encoder/decoder code is performed

- the physical layer blocks use single threaded implementation

To ensure proper behaviour of the integrated simulator a test suite was prepared and successfully performed.

\section{Platform VAlidation}

This section presents description of functional tests performed on physical layer model designed for DAVINCI project. The tests verify that the module behaves as specified and provides the expected interface to the outside world. To ensure a successful implementation (and deployment) of physical layer model for DaVinci project the following tests were designed and performed:

- Successful model deployment on PC/DSP - This test validates TLC scripts (TLC is macro-based language used for mapping models $\mathrm{C}$ code onto target DSP binary) by checking content of files created (by TLC) as an output of DSP deployment process. For different initial conditions, generated output project is checked against compilation

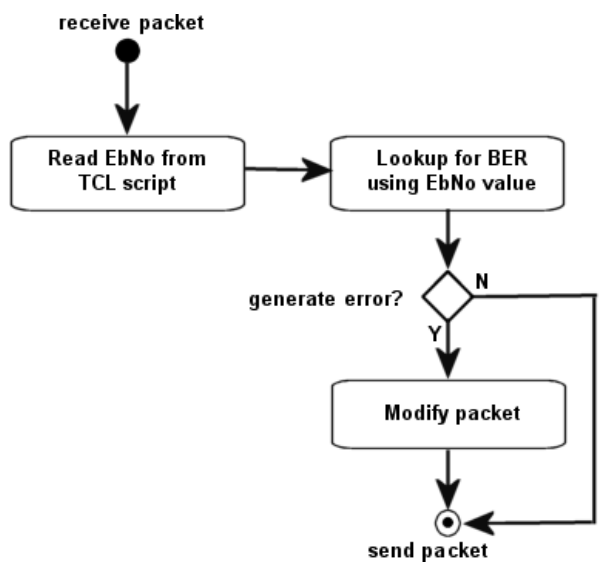

Fig. 7. Introducing frame errors. 
TABLE III

SUMMARY OF THE CHANGES IN THE NS-2 CODE

\begin{tabular}{|c|c|c|}
\hline $\begin{array}{c}\text { Wireless } \\
\text { node } \\
\text { component }\end{array}$ & Changes & Comments \\
\hline $\begin{array}{l}\text { Wireless } \\
\text { PHY }\end{array}$ & $\begin{array}{l}\text { Main packet processing } \\
\text { functionalities switched off; } \\
\text { only sending packets } \\
\text { "up" and "down" }\end{array}$ & $\begin{array}{l}\text { At pressent moment power management } \\
\text { function has been switched off } \\
\text { (no rejecting packets when terminal is off); } \\
\text { power management will be switch on } \\
\text { when simulating mobile terminals. }\end{array}$ \\
\hline Channel & $\begin{array}{l}\text { Replaced by Simulink model; } \\
\text { communication - TCP/IP }\end{array}$ & $\begin{array}{l}\text { The "channel" class is responsible for } \\
\text { estimating propagation time - that } \\
\text { is why it was replaced by Matlab model; } \\
\text { sending packets to Matlab does not } \\
\text { introduce any additional delay. }\end{array}$ \\
\hline $\begin{array}{l}\text { Radio } \\
\text { Propagation } \\
\text { Model }\end{array}$ & Switched off & $\begin{array}{l}\text { Propagation model is not necessary, } \\
\text { as both propagation on time } \\
\text { and noise are added in Matlab model. }\end{array}$ \\
\hline
\end{tabular}

errors and common variables/constants proper initialization.

- Iterative simulation on PC/DSP - This test validates the short term model stability. For different signal inputs test verifies if model returns output signal during 10 subsequent repetitions of a simulation.

- Model output validation for different input signals (DSP/PC) - This test validates model response for different signal inputs. The output is checked with output collected from the legacy Simulink simulation.

Model validation tests were prepared to assure that fully operational model can be successfully deployed both on PC and DSP. On the other hand the separate test suite was introduced to validate outputs of each radio model blocks separately. This group of tests aims at verifying if each of the modules can be deployed separately without compilation errors:

- nb-LDPC encoder output validation

- AWGN output validation

- nb-LDPC decoder output validation.

Additional proof of properly set up of physical layer are valid BER, CWER and FER characteristics, gathered during simulation, compared with those gathered during monte carlo simulation for nb-LDPC stand-alone application.

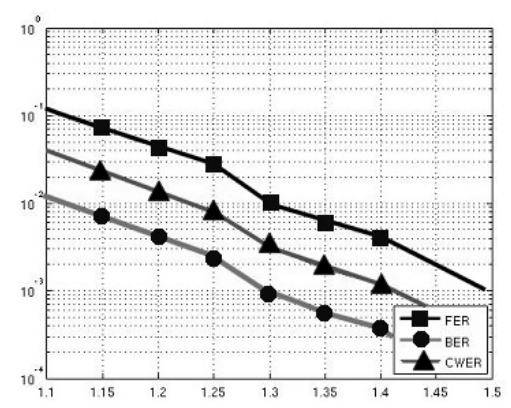

Fig. 8. Results for nb-LDPC codes for original code.

\section{MeAsurements/RESUlts}

As preliminary verification of our integrated platform, following measurements were made with the use of TI DSK c6455:

- Test 1: packet transmission time from ns-2 PHY and back (communication through interfaces IA1, IB see figure Fig. 5), model used default 802.16-2004 Matlab model, packet size - 100B

- Test 2: profiling individual PHY blocks in default 802.162004 Matlab model running on DSK c6455 target board

Also, measurements of Matlab platform were conducted for different hardware configurations:

- Test 3: code profiling (Intel Celeron $2.4 \mathrm{GHz}$ and Intel Centrino, $2.0 \mathrm{GHz}$ Core Duo)

- Test 4: Matlab code's performance evaluation on different target systems.

The measured delay of a packet sent from terminal simulated on Host1 (Test1) through PHY layer (Matlab-DSP) to other terminal and back is on average 100ms (PHY terminal 1 PHY terminal 2). Computing time between terminal's application layers adds another $100 \mathrm{~ms}$. Test2 shows, that in absence of channel coding and with no Ethernet communication, profiled processing time of radio blocks running on target board varies between 5-10ms (for basic WiMAX elements FEC, modulator, OFDM, FFT).

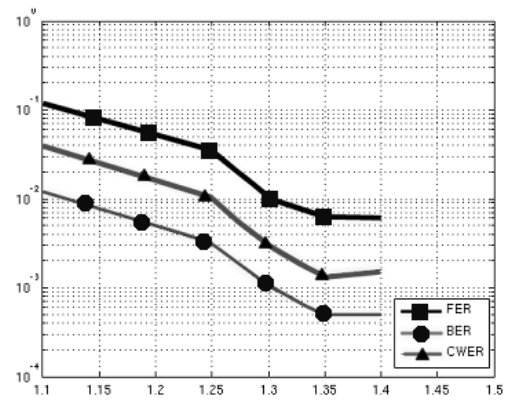

Fig. 9. Results for nb-LDPC codes deployed on DSP board. 


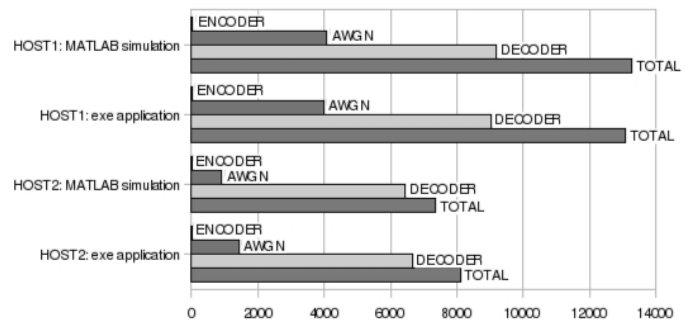

Fig. 10. Performance comparison for two types of Intel processors (PC).

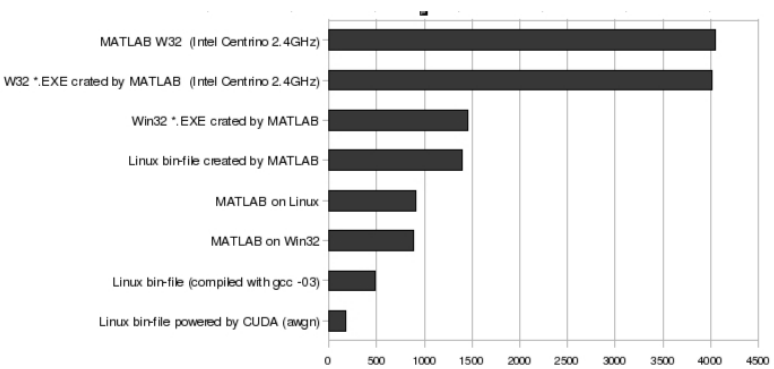

Fig. 11. Matlab code performance on different platforms.

Test 3 focuses on the impact of Gallager's LDPC codes based channel coding on computation times. In this test we were sending $9600 \mathrm{~B}$ packets, and no efforts had been made to optimize decoder. Model was analyzed solely on PC-platform. In the Fig. 10 we should note the fact, that coding decoding time ratio varies

between 1:3 (Intel 2.4GHz, single core, 2GB RAM) and 1:4 (Intel Centrino 2GHz Core Duo, 2GB RAM). Overall decoding time on CoreDuo is smaller by about $40 \%$. The last test scenario (Test4) shows the impact of operating system (Linux/Windows) and model execution method (Matlab model, executable file) on the computation time of AWGN channel (based on 19200bytes sized matrices calculations) - Fig. 11. Moreover we present results achieved by applying NVIDIA CUDA engine to boost computation performance by utilizing processing power of the graphic card board. The results show 16-fold time improvement of Matlab code performance. The test was performed on a computer equipped with GeForce 8600 GT (including 4 on-board processors) and the code was modified to support parallel processing paradigm.

As expected DualCore machine (Fig. 10) achieves much better results than single core processor (see the series 26 from the bottom of the chart).

\section{CONClusions}

In this paper we have presented a novel concept of an integrated WiMAX simulation environment that extends function- ality of legacy ns-2 WiMAX patches by introducing comprehensive Matlab physical layer model in the loop. A prototype has been assembled, by incorporating target board TI DSK c6455 and network communication between nodes running PHY (Matlab/DSP) and upper layers (ns-2). It has been shown that this kind of integration is possible and provides interesting extension of both simulation platforms functionalities in terms of cell level WiMAX simulation. The resulting simulator does not have to pay a cost of a trade-off between fidelity of MAC and physical layer. Using proposed simulator authors plan to perform QoS/QoE and admission control assessment of the target DaVinci link layer including MIMO, OFDM, nbLDPC inside a target WiMAX system according to the ITU-R evaluation requirements for IMT-Advanced candidates.

\section{REFERENCES}

[1] "Requirements related to technical performance for imt-advanced radio interface(s)," International Telecommunication Union, Tech. Rep. ITU-R M.2134, 2008.

[2] A. Belghith and L. Nuaymi, "Design and implementation of a QoSincluded WiMAX module for NS-2 simulator," in the First International Conference on Simulation Tools and Techniques for Communications, Networks and Systems, S. Molnar, J. Heath, O. Dalle, and G. A. Wainer, Eds. Marseille, France: ICST, March 2008, p. 28.

[3] J. Chen, C.-C. Wang, F. C.-D. Tsai, C.-W. Chang, S.-S. Liu, J. Guo, W.J. Lien, J.-H. Sum, and C.-H. Hung, "The design and implementation of WiMAX module for ns-2 simulator," in the 2006 Workshop on $n s-2$. the IP Network Simulator, Pisa, Italy, 2006, article no. 5.

[4] S. Coleri, M. Ergen, A. Puri, and A. Bahai, "A study of channel estimation in OFDM systems," in Vehicular Technology Conference, vol. 2, December 2002, pp. 894-898.

[5] F. da Fonseca, "Description of the wimax module," in the 18th IEEE International Symposium on Personal, Indoor and Mobile Radio Communications, Athens, Greece, 2007.

[6] IEEE Standard for Local and Metropolitan Area Networks - Part 16 Air Interface for Fixed Broadband Wireless Access Systems, IEEE Std. 802.16-2004, Rev. IEEE, 2004.

[7] IEEE Standard for Local and Metropolitan Area Networks - Part 16: Air Interface for Fixed Broadband Wireless Access Systems; Amendment for Physical and Medium Access Control Layers for Combined Fixed and Mobile Operation in Licensed Bands, IEEE Std., 2005.

[8] K.Fall, The ns-2 manual, 2008.

[9] M. Levorato and M. Zorzi, "Performance analysis of type ii hybrid ARQ with low-density parity-check codes," in the Third International Symposium on Communications, Control and Signal Processing, St. Julians, Malta, March 2008.

[10] R. Rouil, "The network simulator ns-2 NIST add-on; IEEE 802.16 model (MAC+PHY)," National Institute of Standards and Technology, 2007.

[11] A. Sayenko, O. Alanen, H. Martikainen, V. Tykhomyrov, A. Puchko, and T. Hmlinen, "Winse: Wimax ns-2 extension," in the 2nd International Conference on Simulation Tools and Techniques for Communications, Networks and Systems, O. Dalle, G. A. Wainer, L. F. Perrone, and G. Stea, Eds. ICST, 2009, p. 46.

[12] Z. Tao, A. Li, J. Zhang, and T. Kuze, "Performance improvement for multichannel HARQ protocol in next generation WiMAX system," Mitsubishi Electric Research Laboratories, 2008.

[13] R. Urban, "Zastosowanie kodow LDPC do poprawy jakosci transmisji w kanale radiowym UKF," in Krajowe Sympozjum Telekomunikacji $i$ Teleinformatyki, Bydgoszcz, 2008, in Polish. 
